PUPT-1325

SHEP 91/92-19

Göteborg ITP 92-20

hep-th@xxx/9206060

\title{
Unitary Matrix Models and 2D Quantum Gravity
}

\author{
S. Dalley ${ }^{1}$, C.V. Johnson ${ }^{2}$, T.R. Morris ${ }^{2}$ and A. Wätterstam ${ }^{3}$ \\ ${ }^{1}$ Joseph Henry Laboratories \\ Princeton University \\ Princeton NJ 08544, U.S.A. \\ and \\ ${ }^{2}$ Physics Department \\ The University of Southampton \\ SO9 5NH, U.K. \\ and \\ ${ }^{3}$ Institute for Theoretical Physics \\ Chalmers Institute of Technology \\ S-412 96 Göteborg, Sweden
}

\begin{abstract}
The KdV and modified KdV integrable hierarchies are shown to be different descriptions of the same 2D gravitational system - open-closed string theory. Nonperturbative solutions of the multi-critical unitary matrix models map to nonsingular solutions of the 'renormalisation group' equation for the string susceptibility, $[\tilde{P}, Q]=Q$. We also demonstrate that the large $\mathrm{N}$ solutions of unitary matrix integrals in external fields, studied by Gross and Newman, equal the non-singular pure closed-string solutions of $[\tilde{P}, Q]=Q$.
\end{abstract}

$6 / 92$ 


\section{Introduction.}

In this letter we shall show how double-scaling limits of unitary matrix models can be interpreted in terms of 2D quantum gravity and its worldsheet expansions. The modified KdV and KdV integrable hierarchies are shown to be different descriptions of the same string theory. Specifically, using the Miura transformation between these hierarchies, and the inverse transformation which we explicitly construct, we map the solutions of the double-scaling limit of symmetric unitary matrix models with $C$ flavours of massless 'quark' [1] [2] to non-perturbative solutions of openclosed string theory in the $(2,2 m-1)$ minimal model backgrounds. The open string coupling $\Gamma$, measured in units of the closed string coupling $\nu$, satisfies $C=1 / 2 \pm \Gamma$ and the boundary cosmological constant is zero. The map implies that the two physical $\tau$-functions of the $\mathrm{mKdV} / \mathrm{KdV}$ hierarchy [3] equal square roots of partition functions of open-closed strings in general. As a simple corollary the continuum limit $1 / N$-expansion of the original unitary matrix model partition function[1] is seen to be nothing other than world-sheets with even numbers of boundaries and $\Gamma^{2}=1 / 4$. The general form of the Virasoro constraints on the $\tau$-function are explained. Correlators of local operators can be evaluated in terms of the flows of the hierarchy in the usual way.

Our analysis also leads to a proof of some transformation equations between solutions of the string equations with open string couplings differing by an integer, generalising a relation proved by Lukashevich, Fokas and Ablowitz[4] for solutions of Painlevé II. Although the Miura transformation from the mKdV hierarchy applies to very general solutions, of particular interest are the unique real non-singular solutions furnished by the unitary matrix models themselves [5][6]. These map to open string generalisations of the non-singular solutions of the $\mathrm{KdV}$ hierarchy ${ }^{1}$ found in [8][9]. Remarkably, the pure closed string partition functions uncovered in those papers are almost surely the solutions determined by Gross and Newman [10][11] for the simplest unitary matrix model in an external field and its formal multicritical generalisations. Damning evidence of this fact will be presented in a later section. The main results are expanded upon in the following section, but first

\footnotetext{
${ }^{1}$ For the case of coupling to the general $(p, q)$ minimal models see also ref.[7]
} 
we conclude this introduction by recalling some salient points about open-closed string theory and matrix models.

Solutions $u\left(z, t_{k}\right)$ of the KdV hierarchy ${ }^{2} \partial_{t_{k}} u=\partial_{z} \mathcal{R}_{k+1}[u]$ must satisfy a couple of physical requirements if they are to represent the string susceptibility of a $2 \mathrm{D}$ gravity theory. Firstly as a function of the dimensionful arguments $\left\{t_{k}, z\right\}$ they must satisfy the renormalisation group $(\mathrm{RG})$ equation for invariance under a change of scale $[8]$, given by $[\tilde{P}, Q]=Q$ in terms of pseudo-differential operators [9] . After some simple manipulation one can rewrite it [8] :

$$
u \mathcal{R}^{2}-\frac{1}{2} \mathcal{R} \mathcal{R}^{\prime \prime}+\frac{1}{4}\left(\mathcal{R}^{\prime}\right)^{2}=\Gamma^{2}
$$

where $^{\prime} \equiv \partial_{z}, \mathcal{R}$ is defined in terms of Gel'fand-Dikii differential polynomials $\mathcal{R}_{k}[u]$ as $\mathcal{R}=\sum_{k=1}(k+1 / 2) t_{k} \mathcal{R}_{k}-z$, and $\Gamma^{2}$ arises as an integration constant. Secondly $u$ must have an asymptotic expansion in $z$ as $z \rightarrow \infty$ which matches that obtained from the perturbative genus expansion of $2 \mathrm{D}$ gravity coupled to some matter theory. For the (flows between the) $(2,2 m-1)$ minimal models coupled to gravity this means that for closed strings $u$ must satisfy $\mathcal{R}=0$ to all orders in the asymptotic expansion. Eqn.(1) has such solutions provided that $\Gamma=0$. Indeed, either $\mathcal{R}=0$ exactly [12] yielding solutions which are complex [13][14] or have singularities somewhere on the flow [15], or one may choose the solution with unique real non-singular flow studied in refs.[8][9][16] (referred to as DJM solution hereafter). $\Gamma$ plays the role of an open string coupling as follows by comparing eqn.(1) with the string equation found by Kostov [17], obtained by performing the double-scaling limit of matrix models with a term $\frac{\Gamma}{N} \operatorname{Tr} \log \left(1+\phi^{2}\right)$ in the potential to generate random holes [18]; each surface is weighted by $\Gamma^{\# \text { holes }}$. In fact Kostov's solutions were restricted to those for which

$$
\mathcal{R}=-2 \Gamma \operatorname{diag}\left\{\left(-\partial_{z}^{2}+u\right)^{-1}\right\}
$$

these obeying $\mathcal{R}=0$ in the $\Gamma \rightarrow 0$ limit. Hence eqn.(1) is more general, encompassing the open string generalisations of the DJM closed string solutions, the previous relation (2) then holding as a matching condition in the sense of an asymptotic expansion as $z \rightarrow \infty$, but being violated at the non-perturbative level. Note that

\footnotetext{
${ }^{2} z$ is essentially the 'space' variable in the $\mathrm{KdV}$ equations, the $t_{k}$ with $k \geq 1$ being generalised time variables.
} 
the renormalised boundary cosmological constant $\rho$ which one may assign to the holes, determined by the position of the branch point of the logarithm, has been set to zero in (1) since this is the case which will interest us in what follows. We shall discuss $\rho \neq 0$ briefly later, in the context of the Virasoro constraints.

Introducing the closed string coupling $\nu$ (renormalised $1 / N$ ) into the string equations (1) and (2) by the rescaling $t_{k} \rightarrow t_{k} / \nu, z \rightarrow z / \nu$, the asymptotic solution is a series in $\nu$ which at the $m$ th critical point takes the form:

$$
u=z^{1 / m} \sum_{g, h=0}^{\infty} A_{g h} \frac{\nu^{2 g+h} \Gamma^{h}}{z^{(2+1 / m)(g+h / 2)}}
$$

$A_{g h}$ is determined in (2) once $A_{00}$ has been fixed, and determined in (1) once $A_{00}$ and the sign of $A_{01}$ have been fixed. For the sake of clarity let us rescale such that $(m+1 / 2) t_{m} \mathcal{R}_{m}=u^{m}+\cdots$, then in the case $z \rightarrow+\infty$ the sphere term is required to be $A_{00}=1$. Using (2) then fixes the disc term to $A_{01}=-1 / m$. We shall also need later the real $z \rightarrow-\infty$ expansion particular to the DJM solution, obtained by taking the new possibility allowed by eqn.(1), $A_{00}=0$ :

$$
u_{\mathrm{DJM}}(z \rightarrow-\infty)=\frac{\nu^{2}}{z^{2}}\left(\Gamma^{2}-\frac{1}{4}\right) \sum_{r=0}^{\infty} \sum_{s=0}^{m r} B_{r s} \frac{\nu^{2 m r} \Gamma^{2 s}}{z^{(2 m+1) r}}
$$

Substituting (4) in (1), the torus and cylinder terms are readily seen to be as above with $B_{00}=1$. The general form then follows from uniqueness together with the symmetry of eqn.(1) (after introducing $\nu$ ) under $\nu^{2} \rightarrow j \nu^{2}, u \rightarrow j u$ where $j$ is any $m$ th root of one.

\section{String Equations and the Miura Map.}

Solutions $v\left(z, t_{k}\right)$ of the modified $\mathrm{KdV}$ hierarchy are defined by the flow equations $\partial_{t_{k}} v=\frac{1}{2} \partial_{z} \mathcal{S}_{k}[v]$ where $\mathcal{S}_{k} \equiv \frac{1}{2} \mathcal{R}_{k}^{\prime}\left[v^{2}+v^{\prime}\right]-v \mathcal{R}_{k}\left[v^{2}+v^{\prime}\right]$. Here $v^{2}$ plays the role of string susceptibility, and its analogous RG equation is just the differential of the unitary matrix model string equation [8] given by the flowing version of the Painlevé II hierarchy:

$$
\sum_{k=1}^{\infty}\left(k+\frac{1}{2}\right) t_{k} \mathcal{S}_{k}[v(z)]+z v(z)=C
$$


( $C$ is again an integration constant). This equation with $C=0$ was first found by Periwal and Shevitz [1] by taking the double-scaling limit of a unitary matrix model with partition function:

$$
Z=\int \mathcal{D} U \exp \left(N \operatorname{Tr} \sum g_{p}\left(U+U^{\dagger}\right)^{p}\right)
$$

The eigenvalues of $U$ lie on the unit circle and the critical point occurs when the support of their large $N$ density function just covers $2 \pi$. The two ends of this support meet to produce $2 m$ zeros when the potential is tuned to the $m$ th critical point. The significance of $C \neq 0$ was later understood by Minahan [2] who derived eqn.(5) by adding a term $\pm \frac{2 C}{N} \operatorname{Tr} \log |1+U|$ to the potential, which may be obtained by integrating out $C$ flavours of bosonic or fermionic 'quarks'.

We will now display a one to one map between solutions of (5) with $C=1 / 2 \pm \Gamma$ and solutions of (1). This map is the Miura transformation $u=v^{2}+v^{\prime}$ familiar in the theory of the $\mathrm{KdV}$ and $\mathrm{mKdV}$ equations ${ }^{3}$. Furthermore we will define a one to one transformation between solutions $v_{C}(z)$ of (5) and solutions of (5) with unit change in the boundary coupling: $v_{C \pm 1}(z)$. Equivalently between $u_{\Gamma}(z)$ and $u_{|\Gamma \pm 1|}(z)$. To avoid confusion we need to state carefully our conventions. The maps apply to general solutions $u_{\Gamma}$ of (1) and $v_{C}$ of (5), not only those obeying the physical boundary conditions discussed below eqn.(2) and below (13). The value of the boundary coupling is used to label any one of the set of solutions with that coupling, and different labels may refer to different functions of the two arguments e.g. setting $v_{ \pm}(z, C \pm 1)=v_{C \pm 1}(z)$ we have $v_{+}(z, A) \neq v_{-}(z, A)$ in general. Finally the label $\Gamma$ for solutions $u_{\Gamma}$ of (1) will always be taken to be non-negative; the actual sign of the open string coupling in the physical solutions is fixed by reference to (2) and will be discussed later.

Let $u(z)$ be a solution to (1) and define

$$
X_{ \pm}[u, v]=\frac{1}{2} \mathcal{R}^{\prime}[u] \mp \Gamma-v(z) \mathcal{R}[u] .
$$

Then $X_{ \pm}[u, v] \equiv 0$ defines a function $v(z)$ (possibly with singularities):

$$
v=\frac{\frac{1}{2} \mathcal{R}^{\prime}[u] \mp \Gamma}{\mathcal{R}[u]} .
$$

3 The authors of ref.[3] used the Miura map in the case $\mathcal{R}=0(\Gamma=0)$ to obtain the Painlevé II hierarchy, but incorrectly dropped the constant $C=1 / 2$. 
Note that one can define $v$ for the $\Gamma \rightarrow 0$ limit of Kostov's solutions by using (2) to cancel $\Gamma$ first. Now

$$
0=X_{ \pm}^{2} \pm 2 \Gamma X_{ \pm}-\mathcal{R}[u] X_{ \pm}^{\prime}=\left(v^{2}+v^{\prime}\right) \mathcal{R}^{2}[u]-\frac{1}{2} \mathcal{R}[u] \mathcal{R}^{\prime \prime}[u]+\frac{1}{4}\left(\mathcal{R}^{\prime}[u]\right)^{2}-\Gamma^{2}
$$

By comparison with (1) we deduce that the inverse transformation is $u=v^{2}+v^{\prime}$, which on substituting in (8) and rearranging gives eqn.(5) with $C=1 / 2 \pm \Gamma$. On the other hand given a solution $v$ of eqn.(5) with $C=1 / 2 \pm \Gamma$, and defining a function $u=v^{2}+v^{\prime}$ we have $X_{ \pm}[u, v]=0$ by rearrangement, and hence (8) is the inverse transformation and $u$ satisfies (9) which is (1).

Thus we have constructed a one to one correspondence between solutions $v_{C}$ of (5) with $C=1 / 2 \pm \Gamma$ and $u_{\Gamma}$ of (1). Since this is true for both choices of $C$ it follows from the Miura map that there is a one to one correspondence between solutions $v_{C}$ and solutions $v_{1-C}$ such that

$$
v_{C}^{2}+v_{C}^{\prime}=v_{1-C}^{2}+v_{1-C}^{\prime}
$$

and by using (8)

$$
v_{1-C}=v_{C}+\frac{2 C-1}{\mathcal{R}\left[v_{C}^{2}+v_{C}^{\prime}\right]}
$$

It is easy to see that this 'flip' transformation changes the sign on boundary conditions for $v$ given by $v \rightarrow \pm z^{1 / 2 m}$ as $z \rightarrow \infty$. On the other hand (5) is odd in $v$ [1] . One can see this for example by induction, using the linear recurrence relation in $\mathcal{R}_{m}$ to prove

$$
v \mathcal{S}_{m+1}^{\prime}-v^{\prime} \mathcal{S}_{m+1}=\frac{1}{4} v \mathcal{S}_{m}^{\prime \prime \prime}-\frac{1}{4} v^{\prime} S_{m}^{\prime \prime}-v^{3} \mathcal{S}_{m}^{\prime}
$$

and noting that since the $\mathcal{R}_{m}[u]$ contains no $u$-independent terms, $\mathcal{S}_{m}$ can contain no terms linear in $v$. Thus, given a solution $v_{C}$ we can construct a solution $v_{-C}$ by

$$
v_{-C}=-v_{C}
$$

Combining this with (10) we obtain the following one to one correspondence between solutions which in particular preserves the above boundary conditions:

$$
v_{C \pm 1}=-v_{C}-\frac{2 C \pm 1}{\mathcal{R}\left[v_{C}^{2} \mp v_{C}^{\prime}\right]}
$$


By using the Miura map we obtain similarly a one to one correspondence between solutions $u_{\Gamma}(z)$ and $u_{\tilde{\Gamma}}(z)$ when $\Gamma \pm \tilde{\Gamma}$ is an integer. It is clear that there are many properties that can be deduced about the solutions of (1) and (5) from these equations. In particular, generalising ref.[4], if one starts with $v_{0}(z) \equiv 0$ one can generate a series of rational solutions to (5) and (1) with $\Gamma-1 / 2$ or $C$ an integer, and finitely many $t_{k} \neq 0$.

Returning to 'physics', if we now re-introduce $\nu$ into (1) and (5) again via $t_{k} \rightarrow$ $t_{k} / \nu, z \rightarrow z / \nu$, one finds at the $m$ th critical point that, when it has an asymptotic expansion, $v$ is of the form

$$
v= \pm z^{1 / 2 m} \sum_{p, q=0}^{\infty} C_{p q} \frac{\nu^{2 p+q} C^{q}}{z^{(2+1 / m)(p+q / 2)}} .
$$

With $t_{m}$ set as below (3), $\left|C_{00}\right|=1$ or $C_{00}=0$. In fact the asymptotics $v \rightarrow \pm z^{1 / 2 m}$ $(v \rightarrow 0)$ as $z \rightarrow+\infty(z \rightarrow-\infty)$ are the ones required by unitary matrix models[1][2]. If $C>0$ the sign choice corresponds to $C$ bosonic (fermionic) quarks[2]. $(C<0$ can be flipped to these by (11) and in the original $C=0$ case[1] the sign is clearly irrelevant). For either sign, a simple calculation reveals that $C_{01}=-1 /(2 m)$ as $z \rightarrow+\infty$, while the leading asymptotic is $v=C \nu / z$ as $z \rightarrow-\infty$. Using the Muira map $\left(u=v^{2}+\nu \partial_{z} v\right)$ on these low orders of perturbation theory one readily finds that the asymptotics map onto those of the DJM solutions as in eqns.(3)(4). Indeed this also determines the sign of the open string coupling to be $\Gamma= \pm(C-1 / 2)$, again the sign choice being that of the $z \rightarrow+\infty v$-asymptotic. This may be used to determine the effect on $\Gamma$ of the maps in (10)-(12); the effect in all cases is to increase or decrease $\Gamma$ by 1 , thus generating one to one maps between solutions with open string couplings differing by integers.

Of particular interest are the known non-singular solutions. When $C=0$ the unitary matrix model (6) provides a unique non-singular flow between the critical points [5][6] having the plus-branch boundary conditions at the $m$ th critical point. Trivially by (11) there are such solutions for the minus branch also. Thus these map under Muira onto DJM-type solutions with non-singular flows, for open string couplings $\Gamma=1 / 2$ and $\Gamma=-1 / 2$. Incidentally for $z \rightarrow-\infty$ (4) implies that $u$ is zero to all orders in the asymptotic expansion, consistent with the $v$-solutions since they also have this behaviour. On the other hand the unique non-singular flows 
of the DJM solutions[16] for $\Gamma=0$ map onto the fermion branch $^{4} v$-solutions with $C=1 / 2$. If these are non-singular, which we expect, then they are also the unique solutions with this property.

Generally, there is to our knowledge no thorough study of the existence and uniqueness of non-singular solutions of the Painlevé II hierarchy with $C \neq 0$ in the literature, nor for eqn.(1) when $\Gamma \neq 0$. Employing the techniques used for the $C=0$ case [5][19] has not yet borne fruit for us. Minahan argued [2] that there should be a unique non-singular real solution for $C>0$ fermionic quarks. These would map smoothly to real non-singular DJM-type solutions of open-closed string theory with $\Gamma<1 / 2$. Due to (11) there would also be a $C<0$ real pole-free solution with positive-branch asymptotic, giving $\Gamma<-1 / 2$. On the other hand similar arguments suggest that there may be unique real non-singular $u$-solutions for $\Gamma<0$.

This two-fold branching when $\Gamma \neq 0$ is also reflected in the $\tau$-functions. As noted in ref.[3], there are for the joint $\mathrm{KdV}$ and $\mathrm{mKdV}$ system related by the Miura map, two tau-functions $\tau_{0}$ and $\tau_{1}$ arising from the two basic representations of the Kač-Moody algebra $A_{1}^{(1)}$. They satisfy

$$
v^{2} \pm \nu \partial_{z} v=-2 \nu^{2} \partial_{z}^{2} \log \tau_{0,1}
$$

respectively and are square roots of partition functions of open-closed string theory in the $(2,2 m-1)$ minimal model backgrounds. The unitary matrix partition function itself (6) is the product $\tau_{0} \tau_{1}$ and so using the fact that $\tau_{0}$ and $\tau_{1}$ have open string worldsheet expansions with $\Gamma= \pm 1 / 2$ respectively $(C=0)$, the continuum worldsheet expansion of (6) has only even numbers of holes and $\Gamma^{2}=1 / 4$. Although it might seem odd that this picture of the continuum limit is not all that similar to that of the $1 / N$ expansion before double-scaling, one must remember that some other matrix model with 'two-cut' behaviour of the eigenvalue density would have sufficed equally [15] . Rather it should caution us that the universal surfaces creamed-off by the double-scaling limit need bear little resemblance to the finite ones we see in the Feynman diagrams. It has been suggested that mKdV describes dense polymer trees [20]. Though such polymers have a 'dual' description as dense selfavoiding loops which is reminiscent of the holes created by open strings, especially

\footnotetext{
4 The sign follows from (8) and by inspection of the $m=1$ numerical solution.
} 
in view of the fact that there is no boundary cosmological constant to suppress their length, we have been unable to make a precise identification. Moreover the authors of ref.[20] were considering the full 2nd mKP hierarchy, including the 'even' time variables. We have been working here with the reduced case obtained by setting these to zero and using only the 'odd' time variables $t_{k}$.

Before closing this section let us mention the Virasoro constraints of openclosed string theory on the tau-functions $\tau_{0}, \tau_{1}$. These are the usual ones of closed string theory except that $L_{-1}$ is apparently missing and $L_{0}$ contains an eigenvalue $\mu$ (as well as the $1 / 16$ ) i.e. $\left(L_{n}-\mu_{i} \delta_{n, 0}\right) \tau_{i}=0$ where $i=0,1$ and $n \geq 0$ [3]. Indeed, substituting $v=\partial_{z} \log \left(\tau_{1} / \tau_{0}\right)$ into the $\mathrm{mKdV}$ flows and using eqn.(5), one readily deduces $^{5}$ that $\mu_{1}-\mu_{0}=C / 2$. Note also that the $L_{0}$ equation is the RG equation for the $\tau$ function and is equivalent to (1) for solutions of the $\mathrm{KdV}$ hierarchy. As remarked previously, for open strings we are free to introduce a constant $\rho$ which weights holes of length $l$ in the worldsheet by $\mathrm{e}^{-\rho l}$. For simplicity the discussion which follows is understood to be perturbative ${ }^{6}$ in $\nu$. We can generate $\tau(\rho)$ from $\tau(0)$ by using the fact that a boundary cosmological constant couples to $L_{-1}[23]$; $L_{-1} \tau(\rho)=\partial \tau(\rho) / \partial \rho$. This equation may be confirmed by a straightforward matrixmodel calculation which determines the r.h.s. as

$$
\frac{\partial u}{\partial \rho}=2 \Gamma \nu \partial_{z} \operatorname{diag}\left\{\left(-\nu^{2} \partial_{z}^{2}+u+\rho\right)^{-1}\right\}
$$

It is then equivalent to $\mathcal{R}=-2 \Gamma \nu \operatorname{diag}\left\{\left(-\nu^{2} \partial_{z}^{2}+u+\rho\right)^{-1}\right\}$, which is always true to all orders in perturbation theory. The general solution is $\tau(\rho)=\mathrm{e}^{\rho L_{-1}} \tau(0)$. This filters through to the Virasoro constraints as

$$
L_{n} \tau(\rho)=\mathrm{e}^{\rho L_{-1}}\left\{\frac{1}{n !}\left(-\rho \operatorname{ad} L_{-1}\right)^{n} \cdot L_{n}+\frac{1}{(n+1) !}\left(-\rho \operatorname{ad} L_{-1}\right)^{n+1} \cdot L_{n}\right\} \tau(0)
$$

where $\operatorname{ad} L_{-1} \cdot L_{m} \equiv\left[L_{-1}, L_{m}\right]$, which on rearranging gives

$$
\tilde{L}_{n} \tau(\rho) \equiv\left[L_{n}-(n+1) \mu \rho^{n}-\rho^{n+1} \frac{\partial}{\partial \rho}\right] \tau(\rho)=0 \quad \forall n \geq-1
$$

\footnotetext{
5 We thank T.Hollowood for bringing this to our attention [21].

6 Amongst other things this obviates the need to burden the reader with certain non-perturbative effects on the boundary cosmological constant which arise for DJM solution (see [7][22] for details).
} 
It is easy to confirm that these $\tilde{L}_{n}: n \geq-1$ satisfy the centreless Virasoro algebra. We do not wish to dwell upon the details here but merely emphasise the obvious but important fact that

$$
\Gamma \neq 0 \Rightarrow \frac{\partial \tau}{\partial \rho} \neq 0 \text { for any } \rho
$$

Thus when $\rho=0$, as we have been considering previously, the constraints at $n \geq 0$ are the previous ones, while $n=-1$ remains there but modified so long as we are dealing with open strings. Therefore $L_{-1}$ is not missing but rather is neutralised as a constraint by the presence of an extra variable other than the $t_{k}$.

\section{Unitary Matrix Model in an External Field.}

In this final section we describe the equivalence of the DJM closed string solution $(\Gamma=0)$ to eqn.(1) and that of the $N \times N$ unitary matrix model in an external field and its formal multi-critical generalisations considered by Gross and Newman[10][11]. This involves the following integral

$$
Z=\int \mathcal{D} U \exp \left(N \operatorname{Tr}\left[U A^{\dagger}+U^{\dagger} A\right]\right)
$$

which can arise in the mean field approach to lattice gauge theory. $A$ is a complex 'background' matrix and some Dyson-Schwinger equations for $Z$ were used in [10] to solve the model in principle to all orders in $1 / N$, the model depending only on the (positive) eigenvalues ${ }^{7} \lambda_{a}$ of $A^{\dagger} A$. The authors noted that the critical behaviour is expressed in terms of the (unscaled) quantities $\sigma_{k}=\frac{1}{N} \sum_{b} \lambda_{b}^{-k / 2}$ for $k=1,3, \cdots$. A special rôle is played by $\sigma_{1}$ since the model has two phases 'strong' and 'weak' according to $\sigma_{1}>2$ or $<2$, the two phases being split by a third order phase transition. Recalling the asymptotic expansions (3)(4) of the DJM solution, precisely this order separates the $z>0$ and $z<0$ spherical free energy, and suggests the identification $z \sim \sigma_{1}-2$, the latter having been identified in ref.[10] as proportional to the 'cosmological constant'. The authors recognized that the simplest critical point in their model is essentially the $m=1$ point of the ordinary

7 It seems likely that they are related in some way to the eigenvalue path integral representation of the DJM solutions [24][8] . 
unitary matrix model (6), which is known to be true also for the $m=1$ DJM solution [8][25] quite apart from the Miura map. This provides some intuition for the coincidence. More immediately it fixes the sign of the identification between $z$ and their cosmological constant to be as above. Note that this implies that $z \rightarrow+\infty$ is their strong coupling regime. The multicritical points in ref.[10] are reached by formally setting $\sigma_{3}=\cdots=\sigma_{2 m-1}=0$; in addition in the continuum limit all $\sigma_{2 n+1}$ with $n>m$ scale to zero. The authors find that there are contributions to all genus in the strong phase, while in the weak phase there are contributions only at genus $g=1 \bmod m$. This agrees with the DJM solution[8] on specializing to an $m$-critical point $t_{k} \propto \delta_{k, m}$ (cf.eqns.(3)(4) with $\Gamma=0$ ). This suggests the identification $t_{k} \sim \sigma_{2 k+1}-2 \delta_{k, 0}$ up to scaling which, given the form of $\sigma_{2 k+1}$ in terms of $\lambda_{b}$, is nothing but a Kontsevich-Miwa transformation[26][11]. This transformation was performed for the unitary matrix model in ref.[11] and we refer the reader there for details. Indeed if we set $t_{k}=\frac{N}{2 k+1}\left(\sigma_{2 k+1}-2 \delta_{k, 0}\right)$ then from eqn.(3.7) of ref.[11] it is clear that their Dyson-Schwinger equations are precisely those of the DJM solution[9]: the Virasoro constraints $L_{n} \tau=0$ for $n \geq 0$. (The $L_{-1}$ constraint is tied up in a similar way to that described in the previous section, due this time not to open strings but to a purely non-perturbative phenomenon which is described further in refs.[7][22][27]. The non-perturbative parameter $\sigma$ which governs the behaviour, corresponding to an eigenvalue representation of the DJM solution on $[\sigma, \infty)$, is zero in the present case (cf. footnote 6 ). The authors of ref.[11] do not in fact take the continuum limit but rather analyse these equations around a topological point. This is not necessary. Our equations of course refer to the case where the continuum limit has already been taken[8] but this does not alter the form of the constraints since they and the $t_{k}$ 's scale homogeneously in the continuum limit.) Finally M.Newman has compared the first few coefficients of the expansions for the multi-critical points of the two theories and found precise agreement [28]. Given all the asymptotic coincidences, the matching Virasoro constraints are persuasive evidence also for non-perturbative agreement, however to completely establish equivalence we would need to show for example that the solutions satisfy the same differential equation(s), together with uniqueness properties given boundary conditions. 
Accepting the equivalence, Gross and Newman's model suggests some interesting interpretations for the DJM solutions. We referred above to the spherical level third order phase transition at $z=0$. However the full non-perturbative solution [9] has no discontinuous transition so that it is in reality at least an infinite order transition, if it is correct to think of this as a phase transition at all. Similar comments have been made in ref.[29]. Rather it has some similarity to a 'roughening' transition for the strong coupling phase world-sheet expansion, a concept familiar in lattice strong coupling perturbation theory[30]. We may think of the point $z=0$ as being the point where the string fluctuations become so large that the concept of these strings breaks down: all world-sheet observables are singular there although the exact theory suffers no phase transition. One might wonder if there is nevertheless some sort of string description in the weak phase. Interestingly this question may be answered by the $m=0$ 'topological' point, a well-defined expansion about a free integral over $U(N)$ identified in ref.[11]. It corresponds to setting all $t_{k}=0$ except $t_{0}$, whence the string equation ${ }^{8}(1)$ (with $\Gamma=0$ ) yields the expected trivial result $u=-1 /\left(4 z^{2}\right)$. It follows that in general the $t_{k} \neq 0$ 'topological expansion' about this point corresponds to the $z \rightarrow-\infty$ asymptotic expansion of (1) (e.g. (4) with $\Gamma=0$ ).

\section{Summary.}

We have shown how the string-theoretic solutions of the $\mathrm{mKdV}$ and $\mathrm{KdV}$ hierarchy are unified by the Miura transformation in the general picture of openclosed string theory, establishing connections between the non-singular solutions of each. It would be interesting to know whether this geometrical picture extends more generally to the KP and mKP hierarchy. We have also presented overwhelming evidence that the unitary matrix model in a tuned external field and its formal multicritical generalisations matches the known non-singular closed string solutions of the KdV hierarchy. It seems likely that lurking behind the results of this letter is a more general setting in terms of quantum gravity, topological gravity and integrable hierarchies.

\footnotetext{
8 The new topological point is simply inconsistent with $\mathcal{R}=0$.
} 


\section{Acknowledgements}

C.J. thanks the S.E.R.C. for financial support. T.R.M. thanks Tim Hollowood

for some enlightening correspondence. S.D. acknowledges discussions with Mike Newman and Andrea Pasquinucci, comments from D.Gross, and is supported by S.E.R.C. post-doctoral fellowship RFO/B/91/9033. 


\section{References}

[1] V.Periwal and D.Shevitz, Phys.Rev.Lett. 64 (1990) 1326, Nucl. Phys. B344 (1990) 731.

[2] J.Minahan, Phys. Lett. B268 (1991) 29, Phys.Lett. B265 (1991) 382.

[3] T.Hollowood, L.Miramontes, C.Nappi and A.Pasquinucci, Nucl. Phys. B373 (1992) 247

[4] N.A. Lukashevich, Diff. Urav. 7 (1971) 1124;

A.S. Fokas and M.J. Ablowitz J. Math. Phys 23 (1982) 2033.

[5] A. Wätterstam, Phys. Lett. B263 (1991) 51.

[6] Č.Crnković, M.Douglas and G.Moore, Nucl. Phys. B360 (1991) 507.

[7] C.V.Johnson, T.R.Morris and B.Spence, Southampton preprint SHEP 90/9130, Imperial preprint TP/91-92/01, hepth@xxx9203022.

[8] S.Dalley, C.V.Johnson and T.R.Morris, Nucl. Phys. B368 (1992) 625.

[9] S.Dalley, C.V.Johnson and T.R.Morris, Nucl.Phys. B368 (1992) 655.

[10] D.J. Gross and M.J. Newman, Phys. Lett. 266 (1991) 291.

[11] D.J. Gross and M.J. Newman, Princeton preprint PUPT-1282, hepth@xxx9112069.

[12] E.Brézin and V.Kazakov, Phys.Lett. B236 (1990) 144;

M.Douglas and S.H.Shenker, Nucl.Phys. B335 (1990) 635;

D.J.Gross and A.A.Migdal, Phys.Rev.Lett. 64 (1990) 127, Nucl. Phys. B340 (1990) 333.

[13] P.G.Silvestrov and A.S.Yelkhovskii, Phys. Lett. B251 (1990) 525.

[14] F.David, Mod. Phys. Lett. A5 (1990) 1019, Nucl.Phys. B348 (1991) 507.

[15] M.R.Douglas, N.Seiberg and S.Shenker, Phys. Lett. B244 (1990) 381.

[16] C.V.Johnson, T.R.Morris and A.Wätterstam, Southampton preprint SHEP 91/92-25 and Göteborg ITP 92-21, hepth@xxx9205056.

[17] I.K.Kostov, Phys. Lett. B238 (1990) 181.

[18] V.A.Kazakov, Phys. Lett. B237 (1990) 212.

[19] H.Flaschka and A.Newell, Comm. Math. Phys. 76 (1980) 65.

[20] Č.Crnković, M.Douglas, G.Moore, Yale/Rutgers preprint YCTP-P25-91 / RU91-36. 
[21] T.Hollowood, private communication (July 1992).

[22] S.Dalley, Mod. Phys. Lett. A7 (1992) 1263.

[23] E.Martinec, G.Moore and N.Seiberg, Phys. Lett. B263 (1991) 190.

[24] T.R.Morris, Nucl.Phys. B356 (1991) 703.

[25] T.R.Morris, FERMILAB-PUB-90/136-T, to appear in Jour. of Class. and Quant. Gravity

[26] A. Marshakov, A. Mironov, A. Morozov, Phys. Lett. B274 (1992) 280.

[27] S.Dalley, C.V.Johnson and T.R.Morris, Nucl. Phys. B (Proc. Suppl.) 25A (1992) 87, Proceedings of the workshop on Random Surfaces and 2D Quantum Gravity, Barcelona 10-14 June 1991.

[28] M.Newman, private communication (February 1992).

[29] J. Ambjørn, C.V. Johnson and T.R. Morris, Nucl. Phys. B374 [FS] (1992) 496.

[30] C. Itzykson, M.E. Peskin and J.B. Zuber, Phys. Lett. 95B (1980) 259;

A. Hasenfratz, E. Hasenfratz and P. Hasenfratz, Nucl.Phys. B180[FS2] (1981) 353 ;

M. Lüscher, G. Münster and P. Weisz, Nucl.Phys. B180[FS2] (1981) 1. 